\title{
EDITORIAL
}

\section{A step in the right direction}

Despite the pressing need for new antibiotics in order to avoid a public-health crisis, antibacterial RED has dwindled seriously in recent years, in part owing to a lack of incentives for companies to make investments in this field. So, the recent passing of an amendment to US legislation that will provide new incentives for antibacterial RED is to be welcomed.

In early May, a bill to reauthorize the Prescription Drug User Fee Act (PDUFA) - through which companies pay fees to the FDA to provide resources to facilitate the timely review of submissions for drug approval — was passed by the US Senate (see the news story on p421-422 of this issue). As widely expected, measures intended to give the FDA greater powers to ensure the safety of marketed drugs feature prominently in the latest version of the PDUFA legislation. For anyone concerned about the shortfall in new drugs to tackle the rising tide of antibiotic resistance, however, the passing of a lowerprofile amendment to this legislation that introduces fresh incentives to spur new antibacterial R\&D could well have been the most welcome aspect.

This 'Antibiotic Safety and Innovation' amendment, which was put forward by Senator Orinn Hatch, includes several provisions related to the development of antibiotics to tackle drug-resistant infections, such as exclusivityrelated incentives for developing known drugs for novel antibacterial indications. Furthermore, as well as reauthorizing the grants and contracts programme for the Orphan Drug Act, which provides various incentives for the development of drugs for diseases that affect less than 200,000 people in the United States, it directs the FDA to convene a meeting to clarify which drug-resistant infections could qualify for orphan drug designation.

The passing of the Hatch amendment represents an important success for organizations such as the Infectious Diseases Society of America (www.idsociety.org), which has been pressing for action to address the shortfall in antibacterial R\&D for years. However, in addition to the lack of market incentives, there are other important issues contributing to this shortfall. Several of these were illustrated in an excellent session at the BIO 2007 conference in May, in which experts in the treatment of bacterial infections and the development of novel antibacterials highlighted some key concerns.

Among these concerns was a rise in the prevalence of bacteria that are increasingly resistant to vancomycin, which has long been a 'gold-standard' treatment for some of the most serious hospital-acquired infections. This rise in resistance has been fuelled by its increasing use. However, although a small number of new drugs for such infections have been introduced that might be more effective than vancomycin in some cases, they are only used relatively rarely - data presented suggested that vancomycin was still used in $\sim 75 \%$ of relevant cases.

This may be partly due to a desire to preserve the effectiveness of the newer drugs in the face of the likely development of resistance. However, a major reason could be that vancomycin is generic, and so cheaper than the newer options. If this is the case, as well as potentially compromising patient treatment, such decisions could be a false economy; if it takes more time in hospital for an infection to resolve with a less-effective drug, this could result in significantly greater costs overall than providing a newer drug that cures the infection faster. In addition, reluctance to use new drugs, and the consequent lack of return on investment for the company producing the drug, has been a significant reason for companies abandoning antibacterial R\&D. So, collecting evidence to allow the formulation of improved guidance on the optimal use of available drugs (ideally aided by more rapid diagnosis), which balances treatment outcomes and all associated costs appropriately, seems desirable.

A presentation discussing vancomycin also highlighted another key issue for developers of antibacterials: the dramatic increase in the amount of clinical trial data that is now needed to support regulatory approval. Back in the 1950s, when vancomycin was first tested, FDA approval was granted on the basis of evidence collected from a few open-label studies that typically involved less than ten patients. By contrast, trials for the recently approved antibiotic tigecycline involved more than 8,000 patients. Although safety standards are now higher, there is a clear need for developing clear regulatory guidance and strategies that could accelerate the development of new antibacterials for the most problematic infections by allowing smaller, more efficient clinical trials. Indeed, perhaps this could be coupled with the possibility of orphan drug designation for such infections, as implied by the Hatch amendment. Certainly, this amendment is a positive step in stimulating a much-needed revival in antibacterial drug discovery and development, but if a public health disaster is to be averted, it must be hoped that it is the first of many. 